\title{
Repensando la universidad europea
}

\author{
María Gómez y Patiño*
}

\begin{abstract}
Resumen
Este texto pretende exponer la génesis, la evolución y el estado actual de la universidad europea, tras haber creado el Espacio Europeo de Educación Superior (EEES) que sigue su andadura evolutiva hasta conseguir los estándares de excelencia unificados para toda la universidad europea. Esta convergencia permitirá un intercambio académico y profesional, primero en la comunidad académica con la movilidad de estudiantes y profesores, y posteriormente la movilidad profesional de todos los estudiantes que hayan obtenido su grado, máster o doctorado en cualquier universidad europea. El proceso tiene beneficios muy notables, así como algunos inconvenientes, lo que supone un reto académico cuyos efectos empiezan a poder percibirse ya en la comunidad académica internacional y hasta mundial.

Las conclusiones y sus interpretaciones permitirán adentrarse en las herramientas pedagógicas y en las actitudes personales.
\end{abstract}

Palabras clave: Convergencia europea, Innovación docente, EEES, Movilidad académica y excelencia.

\begin{abstract}
This essay attempts to describe the genesis, evolution and present situation of the European university after the European Higher Education Area (EHEA) was created and that is still in evolution to get the standard criteria of excellence for the whole European university. This convergence will allow an academic and professional interchange, at the academic community first, with the mobility and interchange of students and professors, and with the professional mobility of all the postgraduates which have got their degree, master or doctorate in any European university. This process has considerable benefits as well as some inconveniences, that is an academic challenge whose effects are now becoming visible either at the international or at the world university community.

The conclusions and their interpretations will allow creating the pedagogic instruments and the personal attitudes.
\end{abstract}

Key words: European convergence, Teaching Innovation, European Higher Education Area (EHEA), Academic Mobility and excellence.

* Nacionalidad: española. Dra. en Sociología. Universidad de Zaragoza, Dpto. Psicología y Sociología. Doctor Cerrada, 1-3. 50005 ZARAGOZA - ESPAÑA. Tel. 00 349767646 48. Fax. 00349767620 03. e-mail: mariagp@unizar.es 


\section{A modo de introducción}

El Espacio Europeo de Educación Superior (EEES) es la formulación de una respuesta coordinada e institucional a un nuevo contexto económico, social y académico.

La clave es la necesidad de adaptación a una nueva realidad académica y social. La iniciativa de las autoridades comunitarias podría entenderse como un intento de que Europa no se quede atrás en un contexto académico internacional y globalizado donde los cambios se suceden a gran velocidad. La necesidad de cambio venía impuesta por el proceso de internacionalización que las universidades han iniciado. Esta necesidad se sentía, antes incluso de la Declaración de Bolonia, por lo que no se parte de cero. De hecho gran parte de las universidades tenían ya numerosas experiencias que se aproximan al nuevo modelo.

Como en muchos otros ámbitos de la vida, lo académico no se podía sustraer a los cambios: la sociedad iba por delante de la Academia. Los profesionales europeos tenían el derecho a prestar sus servicios profesionales en el país europeo que quisieran, pero eso no estaba resuelto, ni unificado, desde el punto de vista de la formación académica.

Según Sanagustín et al. (2004:67), el EEES es el reflejo de la integración a nivel educativo que se pretende llevar a cabo en la Unión Europea, para lo que los programas Erasmus (1987-1994) y Sócrates-Erasmus (1995-2006) de movilidad de estudiantes han dado un gran impulso.

Lo cierto es que el EEES, además de generar un espacio unificado europeo, está modificando las prácticas profesionales y académicas no sólo en el espacio europeo, sino que trasciende al continente y le permite entrar en un proceso de internacionalización académica.

\section{Objetivos}

El EEES, que persigue cinco grandes objetivos: 1) facilitar la homologación y el reconocimiento de títulos en la Unión Europea; 
2) facilitar la formación de quienes ya trabajan; 3) promover la movilidad de estudiantes y profesores, 4) acercar el perfil de la formación a las necesidades reales de los empleadores; y 5) construir una universidad europea competitiva frente al modelo americano, ha de acometer un gran reto, que con las resistencias personales/profesionales previsibles, habrá de superar. No se trata de tener los mismos planes de estudio en todos los países que lo suscriben, sino de que se usen los mismos criterios con el objetivo de que las titulaciones sean comparables, y preferiblemente homologables.

1) Uno de los grandes cambios que el EEES propone es que los títulos universitarios ya no pueden ser un pliego de papel que sirva de adorno para el salón o el despacho profesional. A partir de ahora hora irán acompañados del Suplemento al Título, en el que se hará constar qué se ha estudiado, qué competencias se han adquirido y a qué salidas laborales se puede acceder.

2) El EEES es un instrumento fundamental para facilitar el proceso de internacionalización de las universidades. Siendo la comunidad universitaria más consciente de que una enseñanza superior de calidad se encuentra ligada a una mayor movilidad de estudiantes y personal docente. Esto se potenciará de manera decisiva con los nuevos instrumentos.

3) El EEES es un modelo universitario en vigor, soportado por un entramado legal muy complejo. La Universidad española tiene la obligación de aplicar las leyes en vigor en el marco de su autonomía universitaria, donde su incumplimiento no es una opción. Se trata de la nueva realidad de la universidad española y se encuentra en pleno proceso de adaptación e implantación.

4) El plazo de adaptación ha durado hasta el curso 2010/2011 y en este curso se han de convertir las antiguas licenciaturas y diplomaturas en los nuevos grados. Todas las universidades españolas han iniciado ya el proceso. Algunas, incluso, ya tenían todos sus títulos adaptados en 2009. Gran parte de 
las universidades espera tener adaptados las enseñanzas de Grado, de Master y de Doctorado.

El soporte documental de todas estas modificaciones ha sido aportado por el consenso sobre el que apoyar estas ideas y objetivos han generado inmensas cantidades de papel, habiéndose creado legislación en todos los países firmantes, y ratificándose infinidad de documentos. Sanagustín/Cano (2005).

\section{Configuración, legislación, convenios y planes}

En el EEES existen (según el RD 1393/2007) tres tipos de titulaciones: Grado, Master y Doctorado: 1) El Grado proporciona una formación general en una o varias disciplinas, orientada a la preparación para el ejercicio de actividades de carácter profesional; 2) El Master persigue que el estudiante adquiera una formación avanzada, de carácter especializado o multidisciplinar, orientada a la especialización; y 3) El Doctorado tiene como finalidad la formación avanzada del estudiante en las técnicas de investigación e incluirá la elaboración y presentación de la correspondiente tesis doctoral (RD. 1393/2007 Arts. 9, 10 y 11, respectivamente).

El Grado sustituye a las Licenciaturas, las Diplomaturas, las enseñanzas técnicas y el $2^{\circ}$ Ciclo. En lo esencial equivale a las licenciaturas desde la perspectiva de la formación y los contenidos. En el caso de las licenciaturas de cinco años, el Grado no implica un descenso en la calidad y la cantidad de los estudios, sino una reorganización de los mismos. Este cambio de cinco a cuatro años ya ha venido sucediendo para muchas licenciaturas con motivo de la Reforma que se llevó a cabo en 1993, sin que ello supusiera una merma en la valoración de los estudios, y se seguían llamando Licenciaturas. Supone una ampliación de uno y dos años de estudios respecto de las diplomaturas, las ingenierías técnicas, la arquitectura técnica y las titulaciones de $2^{\circ}$ Ciclo. Sólo estarán fuera de esa duración los grados en Farmacia (5 años), Arquitectura y Medicina (6 años). 
El Master no es comparable con ninguna enseñanza oficial anterior que existiera en la Universidad. Lo más parecido son los llamados Títulos Propios de la Universidad, que son enseñanzas encaminadas a completar la formación universitaria mediante la actualización y especialización en cuestiones científicas, profesionales, artísticas y culturales demandadas por el entorno social. En función de los requisitos de acceso y la carga lectiva, hay tres tipos: Master, Experto y Especialista.

El Doctorado equivale a lo que anteriormente llamábamos Doctorado o Estudios de tercer Ciclo. Las enseñanzas de Doctorado tienen como finalidad la formación avanzada del estudiante en las técnicas de investigación. Culmina con la obtención del Título de Doctor tras la defensa de una Tesis Doctoral.

Hay sin embargo dos diferencias importantes. Antes el periodo de formación se dividía en dos años; el primero era de docencia y suponía 20 créditos; el segundo era de investigación y se valoraba en 12 créditos. Terminados los 32 créditos, el Doctorado se culminaba con la defensa de la tesis doctoral. Ahora, en el EEES, para obtener el título de Doctor es necesario haber superado un periodo de formación y un periodo de investigación organizado. El periodo de formación consiste en la realización de 60 créditos ECTS de Master (realizados bien en un solo master o en varios). Tras este periodo se accede a la investigación que consiste en un conjunto organizado de actividades formativas y de investigación y que culmina con la presentación de la Tesis Doctoral. En este periodo combinarán seminarios, fundamentalmente metodológicos, y actividades de investigación, pudiendo vincularse a uno o más estudios de Master. El EEES no invalida de ninguna manera los títulos existentes otorgados antes de su implantación.

\section{Planteamientos nuevos}

Esta reestructuración académica europea trae aparejados cambios sustanciales no sólo en los contenidos, sino también en los 
continentes. Cambian los actores, sus roles y necesariamente sus actitudes. La institución académica sigue siendo el sustento de medios pedagógicos y tecnológicos que proporciona los espacios y los materiales necesarios tanto para estudiantes como para profesores. Ha de servir de apoyo y de estímulo a la vez que de base para cualquier implementación o cambio académico. Ha de pasar de ser una institución estática y rígida a realizar una gestión dinámica tanto de los recursos económicos, financieros, humanos y tecnológicos de primer orden.

4.1 El profesor/docente que era el actor que administraba el conocimiento, evaluaba y calificaba, ha de cambiar también su rol. Ya no administrará el conocimiento teórico sino que se convertirá en una especie de tutor o facilitador, cuya principal misión será el enseñar al estudiante a gestionar su propia dinámica, tanto para establecer sus contenidos, como el tiempo de dedicación a la materia, como a conseguir las competencias personales y profesionales que le permitirán adquirir unas destrezas personales. De alguna forma, deberá enseñar al estudiante a aprender. El estudiante va a tener menos tiempo de formación en su grado, por lo que le convendrá adaptar mejor sus contenidos a su práctica profesional futura y por otra parte, deberá familiarizarse con el aprendizaje permanente (lifelong learning) o con el aprendizaje autónomo, sin la presencia del profesor o del tutor. Finalmente, el profesor deberá evaluar y calificar al estudiante al que previamente habrá adiestrado en la forma de evaluación y superación de su asignatura.

El profesor deberá ser capaz de enseñar a aprender este proceso en su totalidad. De forma muy clara, Zabalza (2004) resume los cambios más importantes que se están produciendo en el contexto universitario actual, señalando las siguientes ideas descriptivamente:

- Una docencia centrada en el estudiante requiere capacitarlo para el aprendizaje autónomo y dotarlo de herramientas para el estudio. 
- Un diferente papel del profesor, que pasa, de estar centrado en los contenidos de la materia, a ser gestor del proceso de aprendizaje de los alumnos.

- Una organización de la información orientada a la consecución de competencias (competencias generales para todos los estudiantes y competencias específicas de cada titulación).

- Cambios en la organización de los aprendizajes: una perspectiva curricular del trabajo del docente que refuerce la continuidad y la coordinación.

- Una nueva definición del papel formativo de las universidades: la formación a lo largo de la vida. Esto implica que no todo lo que puede o debe ser enseñado en cada materia para el desempeño de una profesión ha de ser enseñado, necesariamente, en los años universitarios.

- Un nuevo papel de los materiales didácticos, que pasarán a ser recursos capaces de generar conocimiento de alto nivel y de facilitar el aprendizaje autónomo. En este sentido las Técnicas de la Información y la Comunicación juegan un importante papel.

4.2 El estudiante debe pasar a ser el centro del sistema educativo, como principal actor del mismo. Para ello se valorará el esfuerzo que el estudiante necesita realizar para superar las enseñanzas, no únicamente las horas de clase presencial, sino también a cambiar la filosofía de éstas, otorgando más importancia al manejo de las herramientas de aprendizaje que a la mera acumulación de conocimientos. El propio estudiante debería estar preparado para autoevaluarse de acuerdo con los objetivos tanto de la asignatura o de la carrera, rectificando los posibles errores de aprendizaje iniciales. Todos los cambios en el proceso de aprendizaje-evaluación han de ser gestionados por el estudiante, tutorizado por el profesor, que a partir de la implantación completa del Plan Bolonia, se convertirá en un valiosísimo consejero-asesor-evaluador. En este sentido, Martínez Segura (2009:27) afirma que en el nuevo espacio de aprendizaje autónomo, el estudiante asume una 
parte importante de la responsabilidad en la organización de su trabajo ajustándolo a su propio ritmo, para lo que requerirá la tutorización del profesor, una mayor preparación por su parte que le permita transferir información entre diferentes aprendizajes y disponer de una infraestructura tecnológica y didáctica adecuada. Veáse Gosling (2002).

\section{Nuevos Métodos de aprendizaje-enseñanza- evaluación}

El EEES implica la instauración de nuevas metodologías docentes, en detrimento de las tradicionales clases magistrales. Se convertirá por tanto, en: 1) Evaluación continua: seguimiento diario al trabajo personal del alumno mediante evaluaciones continuas. Para llevar a cabo la evaluación continua se proponen principalmente dos herramientas: el uso de todas las posibilidades que ofrece Internet, las nuevas tecnologías TIC y las tutorías personales; y Enseñanza práctica: intervención activa del alumno a través de ejercicios, trabajo en grupo, prácticas profesionales, etc.

\subsection{Aprendizaje permanente (Life-long-learning - LLL)}

El European Qualifications Framework (EQF) es una recomendación a los Estados miembros que establece el año 2010 como fecha límite para que se realicen los ajustes entre sus sistemas de calificaciones y el EQF, y el 2012 como fecha de aplicación completa. Es una herramienta para fomentar el llamado aprendizaje permanente (Life-long-learning-LLL). Su objetivo es vincular los diferentes sistemas nacionales de calificaciones mediante ocho niveles de referencia comunes a toda Europa y que abarcan desde los certificados de educación escolar hasta el doctorado.

\subsubsection{El Portafolio y el PDP (Personal Development Planning)}

Para este proceso de cambio metodológico integral, el uso del Portafolio del Estudiante, se convierte en una herramienta no sólo útil, sino muy necesaria, como señala Hilda E. Quintana (2000) 
con las adaptaciones propias de cada disciplina, tal como señalan White (1989), Tierney (1991), o Barberá (2005). Es destacable el papel que le atribuyen Fernández y Maiqués (2001) al portafolio, ya que para ellos es el profesor el que tiene el derecho y la responsabilidad de demostrar su profesionalidad, por lo que el portafolio no sólo será una herramienta discente sino también docente. Permite la auto o hetero-evaluación. Este instrumento está íntimamente relacionado con lo que se conoce en el mundo anglosajón como Personal Development Planning (PDP) que consiste en esta planificación del tiempo por parte del estudiante (Gosling, 2002).

Asimismo, la herramienta portafolio electrónico puede utilizarse por tanto como tecnología digital y como parte tanto del proceso presencial y también del e-learning, con el objeto de coleccionar las múltiples evidencias del proceso de aprendizaje en diferentes medios (audio, video, gráficos, textos), por lo que aporta una gran diversidad de expresión. El lenguaje multimedia aporta posibilidades que no estaban presentes antes de la aplicación de las nuevas tecnologías en la docencia universitaria.

\section{Planes de Mejora e Innovación de la Docencia}

Todos los Vicerrectorados de la universidad, ya sea el de Ordenación Académica, como el de Investigación o el de Personal Docente e Investigador son conscientes de la necesidad, más que de la conveniencia, de modificar la forma de pensar y la mentalidad de la comunidad universitaria. De ahí que gran parte de las universidades preocupadas por los cambios que se están produciendo, pretenden adaptar necesidades con expectativas y éstas con mejoras en la innovación docente. Fundamentalmente serán los profesores los encargados de transmitir cada cambio a los estudiantes en particular, pero también a la sociedad, en general, de la modificación tanto en la mentalidad de los miembros universitarios como la de los ciudadanos europeos.

En el Plan de Mejora e Innovación Docente 2004-09 (ICE-2004:910) estos programas se articulan alrededor de tres elementos que 
hoy son imprescindibles para definir una docencia de calidad en el nuevo marco social y educativo europeo. El primer elemento es la competencia pedagógica del profesorado ${ }^{1}$, comprometido con una docencia centrada en el logro de competencias profesionales y en el proceso de aprendizaje de los estudiantes. Por ello la formación del profesorado es un componente básico del plan. El segundo elemento es la innovación docente, condición necesaria para el logro de la calidad y la mejora continua. Finalmente se resalta el apoyo a los estudiantes, con acciones diversas pero, sobre todo, con planes de acción tutorial, promovidos y coordinados desde los diferentes centros.

\section{Nuevas competencias}

La base competencial pedagógica de los profesores es crítica para mejorar la calidad de la docencia y adaptarla al Espacio Europeo de Educación Superior. Han de ser preferentes, según el ICE (2004:26), las siguientes competencias:

a) competencias meta-cognitivas, que le conviertan en un profesional reflexivo y auto-crítico con su enseñanza, con el objetivo de revisarla y mejorarla de forma sistemática.

b) Competencias gerenciales, vinculadas a la gestión eficiente de la enseñanza y de sus recursos en diversos ambientes y entornos de aprendizaje.

c) Competencias sociales, que le permitan acciones de liderazgo, de cooperación, de persuasión, de trabajo en equipo, etc., favoreciendo así la formación y disposición de sus estudiantes en este ámbito, así como su propio desarrollo y acción profesional.

d) Competencias afectivas, que aseguren unas actitudes, motivaciones y conductas favorecedoras de una docencia comprometida con los nuevos planteamientos.

Asimismo, según el ICE (2004:27-8), entre los grandes cambios conceptuales en el proceso de enseñanza-aprendizaje, que afecta tanto a profesores como estudiantes, son destacables:

1 Las cursivas son de la autora. 
- Conocimiento del proceso de aprendizaje del estudiante en contextos académicos y naturales.

- Planificación de la enseñanza y de la interacción didáctica.

- Utilización de métodos y técnicas didácticas pertinentes

- Gestión de interacción didáctica y de las relaciones con los alumnos

- Conocimiento de normas legales e institucionales reguladoras de derechos y deberes del profesor y del estudiante.

- Gestión de su propio desarrollo profesional como docente. Asimismo, la innovación, en métodos pedagógicos, evaluación, etc., debe ser controlada, evaluada y revisada, porque la innovación por sí sola, no es un signo de calidad; lo es si facilita, incrementa y mejora el aprendizaje de los estudiantes y el logro de los objetivos de la titulación.

Se crean planes y programas de formación, orientación y acción tutorial, una serie de actividades de formación en dos direcciones:

- Ofrecer al profesorado cursos y seminarios sobre temas de especial relevancia que inciden en cuestiones metodológicas para la mejora del proceso de enseñanza y aprendizaje, y

- Dar respuesta a las peticiones formuladas por los Centros, los Departamentos o los grupos de profesores, relacionados con aspectos concretos de su labor docente.

La realidad apunta como necesaria la figura de un profesor académicamente potente, experto, maduro, capaz y brillante, sin decir por ello que el anterior profesor no lo fuera, del profesor universitario se esperan infinidad de virtualidades, competencias y capacidades. Son asimismo numerosos los autores que van describiendo todas estas potencialidades, entre los que se citará por ejemplo a González Ramírez (2005), quien ya estaba pensando en términos del profesor universitario europeo dentro del EEES. En realidad, el perfil del profesor excelente, ha sido definido por otros muchos autores, antes incluso de la aparición del EEES, como es el caso de Ramsden (1992), quien, de 
forma similar a otros autores, describe a un ser absolutamente comprometido y entregado y una gran capacidad de adaptación e innovación. Ni se menciona (se da por supuesto) que se espera de él una alta competencia en la disciplina impartida y gran capacidad de gestión de su propio conocimiento, pero deberá saber seleccionar y preparar los contenidos, planificando el proceso de enseñanza-aprendizaje. Las explicaciones que ofrezca deberán ser comprensibles, bien organizadas y actualizadas, describiendo los conceptos relevantes. Sería deseable que centrara su atención en los conceptos más relevantes para favorecer la transferencia y aplicación de lo aprendido a otros contextos, partiendo de lo que el estudiante conoce y enlazándolo con otros.

Muy adecuado sería que supiera diseñar las metodologías y las estrategias más convenientes, y que con menos recursos tuviera mayor impacto. Su comunicación con los estudiantes debería ser muy positiva tanto a título individual como grupal, autorizando a sus propios estudiantes de una forma personalizada, manejando con destreza las Tecnologías de la Información y la Comunicación (TIC), siendo capaz de evaluar el aprendizaje y reflexionar sobre la enseñanza lograda, modificando e innovando los aspectos menos positivos, identificándose con la institución, colaborando con sus colegas, siendo exquisito en el cumplimiento de la ética de su profesión.

\section{Discusión y Debate}

El Espacio Europeo de Educación Superior (EEES), es posiblemente la mayor revolución universitaria en el viejo continente. Como cualquier cambio o innovación, tiene infinidad de seguidores fieles y de detractores críticos, por lo que parece necesario introducir algunas voces disonantes, que son muchas y muy importantes. No obstante, si el EEES pretendía mejorar las condiciones y la situación laboral interna de los docentes, así como la calidad e incluso la excelencia académica, quedarían muchos puntos por revisar que no han sido tratados y que afloran desde distintas voces críticas, que han de ser debatidas. Quizá el texto más impor- 
tante producido con un espíritu crítico ha sido el conocido como Informe Tuning, libro coordinado por Julia González, profesora de la Universidad de Deusto (España) y Robert Wagenaar, profesor de la Universidad de Groningen (Holanda) en la que han participado docenas de profesores de todas las universidades europeas, al que se hará referencia en este apartado, pero las críticas tanto en radio, televisión, prensa, o en manifestaciones callejeras han sido innumerables, por lo que sólo se podrán comentar algunas de ellas.

\subsection{Algunas Críticas:}

\subsubsection{Falta de democratización}

Este gran cambio en la universidad europea hace aflorar en ocasiones, vicios que estaban ocultos $u$ otros nuevos que nacen al calor de la crisis, del cambio, y en ese sentido, esta cita del artículo de un catedrático de la Universidad de Barcelona habla por sí solo:

\subsubsection{1 "Lo peor de cada casa o Bolonia como excusa (EL PAÍS 1-12-2008)}

No pretendo abrir aquí un debate sobre la meritocracia o sobre la democracia censitaria, pero constato que, en contra de lo que a primera vista podría parecer (y resultaría deseable), la presunta democratización en el acceso a los cargos ha provocado la generalización de procedimientos dudosamente democráticos, lo que en el caso de la elaboración de los nuevos planes de estudio se ha concretado en la designación, por parte de las autoridades académicas, de comisiones pretendidamente técnicas que terminaban decidiendo acerca de cuestiones de contenido a uña de caballo, eliminando asignaturas o proponiendo otras nuevas, sin dar ocasión a que tuviera lugar un debate abierto, en el que pudieran participar todos los sectores afectados. Manuel Cruz, Catedrático en la Universidad de Barcelona y director de la revista METROPOLIS." 
Ciertamente, y este problema no aparece sólo en los temas académicos, con demasiada frecuencia la creación e implantación de muchos de los cambios que se producen en la realidad social, no están conducidos de la mano de expertos ni de las personas afectadas, son más bien tecnócratas europeos los que toman las decisiones desde una capital europea, con poco o nulo contacto con la realidad que están tratando de mejorar. No ha lugar para la reflexión y mucho menos para el debate, las decisiones han de tomarse con un plazo y gran celeridad pues los emplazamientos son inamovibles.

Las personas afectadas, cuando conocen las decisiones, sólo pueden adoptarlas o ser críticas con ellas, pero la ley tiene mucho peso y ha de cumplirse. Las adaptaciones personales no son siempre naturales y pueden resultar muy forzadas, con todo lo que ello implica de resistencias psicológicas o incapacidad de adaptación al nuevo sistema. De hecho la universidad española ha facilitado -y lo sigue haciendo- un sistema de prejubilación para aquellas personas que tienen muchos años de docencia, al estilo clásico español, y que no pueden o no quieren adaptarse a estas innovaciones académicas.

\subsubsection{Falta de financiación}

Otra de las críticas es la falta de financiación europea y nacional para implantar la reforma. Así, en el caso de España, el rector de la Universidad de Sevilla, por ejemplo, ha denunciado que no se especifique en los nuevos planes de estudios de las facultades sevillanas mandados a revisión por la ANECA cómo se va a implantar lo reglado por el Real Decreto en cuestión de número de alumnos por clase y seguimiento del trabajo personal del alumno, puesto que no disponen de medios ni estructuras suficientes. Por parte del alumnado, se ha criticado que la reforma no venga acompañada de un aumento de becas sino del fomento de los llamados préstamos-renta, préstamos públicos ligados a renta futura.

Gran parte de las innovaciones que se pretenden hacer tienen "coste 0 " lo que constituye un serio problema a la hora de 
abordar la enseñanza de calidad. El sistema tutorial y el seguimiento personalizado de los estudiantes aconseja reducir considerablemente la ratio de profesor-por-estudiante, hecho que aún no se ha producido. Ni se ha reducido el número de estudiantes ni aumentado el número de profesores, por lo que no parecen existir demasiadas posibilidades de llevar adelante el proceso con éxito.

\subsubsection{Mercantilización de la universidad pública}

Una crítica importante es la mercantilización de la universidad pública que conlleva la reforma universitaria. Este fragmento es un buen ejemplo:

\subsubsection{1 "La descomposición de la Universidad (EL PAÍS, 10 de noviembre de 2008).}

(...) lo que las autoridades políticas no dicen (...) es que bajo ese nombre pomposo se desarrolla en España una operación a la vez más simple y más compleja de reconversión cultural destinada a reducir drásticamente el tamaño de las universidades -y ello no por razones científicas, lo que acaso estuviera plenamente justificado, sino únicamente por motivos contables- y a someter enteramente su régimen de funcionamiento a las necesidades del mercado y a las exigencias de las empresas, futuras empleadoras de sus titulados; una operación que, por lo demás, se encuadra en el contexto generalizado de descomposición de las instituciones características del Estado social de derecho y que concuerda con otros ejemplos financieramente sangrantes de subordinación de las arcas públicas al beneficio privado a que estamos asistiendo últimamente. José Luis Pardo, catedrático de Filosofía en la Universidad Complutense de Madrid".

Ésta es una de las preguntas más repetidas en los distintos sectores críticos: ¿Son las razones últimas que subyacen en estas operaciones de cambio de carácter científico o financiero? La mera duda/pregunta ya es un grave lastre para la universidad pública. 


\subsubsection{Críticas desde Colegios Profesionales}

Las carreras de Medicina y de Arquitectura siempre han sido las más largas, lo que, con la llegada del grado europeo se convierte en una situación cuanto menos incómoda, cuando no injusta, pues su equiparación pasa por una reducción considerable de su status universitario. Como resultado, los Colegios Profesionales de Médicos y de Arquitectos critican el proceso, ya que devalúa la categoría de sus titulaciones equiparándolas a las diplomaturas. Desde hace algún tiempo se vienen realizando peticiones al gobierno, a través de sus representantes estatales, sin conseguir los objetivos a los que aspiraban. Por lo que se realizaron manifestaciones al efecto, entre ellas la del día 23 de octubre 2009 frente al Ministerio de Educación. Noticia de la que se hace eco el semanal digital2: Los arquitectos y médicos no entienden cómo sus titulaciones se convierten en titulaciones de grado con el nuevo marco legislativo y como en cambio las ingenierías se convierten en titulaciones de Master.

Los colegios profesionales de médicos y arquitectos piden a través de un comunicado del presidente del Consejo Superior, en el Pleno de Consejeros celebrado el día 5 de marzo de 2009, tomó conocimiento de los Acuerdos de Consejo de Ministros que establecen las condiciones a las que deberán adecuarse los planes de estudios conducentes a la obtención de títulos que habilitan para el ejercicio de las profesiones reguladas de Ingeniero e Ingeniero Técnico, publicados en el Boletín Oficial del Estado de 29 de enero de 2009, lo siguiente: "Promover la modificación del acuerdo del Consejo de Ministros de 14 de diciembre de 2007 y de la orden ECI/3856/2007, con el fin de que el Título de Grado que da acceso a la profesión de Arquitecto cuente con un Proyecto Fin de Carrera considerado como práctica profesional, con 60 créditos ECTS y tenga el nivel académico de Master."

Los corporativos profesionales tratan de defender su situación y argumentan que si han tenido que estudiar tradicional-

2 www.digital.com, 24-10-2009. 
mente más años, no parece lógico que ahora se les equipare a una diplomatura.

\subsubsection{Algunas voces muy críticas}

Tal como se ha comentado anteriormente, el Informe Tuning $^{4}$, los críticos al proceso de Bolonia han señalado varias vías por las que se implantaría la mercantilización de la universidad pública:

- Equiparación práctica del horario estudiantil al de un horario laboral debida al aumento de las horas lectivas presenciales obligatorias que se requieren para aprobar la cantidad de créditos necesarios. Se entiende desde los sectores críticos al proceso de Bolonia que el cambio provocará una elitización de la enseñanza universitaria, en cuanto para acceder a ella será necesario disponer de mayores cantidades de dinero sin trabajar para ello, pues el aumento de horas presenciales obligatorias (especialmente en postgrado) dificultaría trabajar y estudiar al mismo tiempo. Por otro lado, estos aumentos en los costes económicos (de tiempo y dinero) que supone la adaptación al EEES, no se compensan con un aumento eficaz de las ayudas y becas nacionales o europeas en este ámbito.

- Profesionalización del estudiante: según sus críticos, en el proceso de Bolonia la universidad se concibe como productora de individuos válidos para introducirse en el mercado laboral europeo. No se trata ya de desarrollar y transmitir conocimiento o incluso conocimiento con aplicación social sino de formar trabajadores. En este sentido cualquier aplicación social del conocimiento va a tener que ser impulsada por em-

3 Se conoce así al libro publicado en 2004 en la Universidad de Deusto, escrito por docenas de profesores europeos críticos con el EEES y resultado de un informe final que fue coordinado por Julia González y Robert Wagenaar (en bibliografía).

4 El término anglosajón Tuning, que en español se usa frecuentemente sin traducir, tiene un doble significado. El primero hace referencia al afinado de los instrumentos, y por extensión, también podría utilizarse como el afinado de la estructura universitaria europea, pero es necesario señalar que el "tuning" en los automóviles, por ejemplo, hace referencia a un makeado o tuneado en términos coloquiales, para indicar que el automóvil ha sido modificado sobre todo externamente, dado que el chasis y su numeración sigue siendo igual, pero se habrá hecho un gran gasto en cambiar la imagen del vehículo. 
presas privadas y, por tanto, presumiblemente motivada por intereses privados.

Cabe plantearse si la homologación del sistema universitario lo ha de estar en cuanto a exigencias o también en cuanto a recompensas (el remunerativo incluido). Los salarios de los académicos centro-europeos multiplican por cuatro el salario de un académico español, portugués o griego. Las críticas son de tal calado que no pueden ser ignoradas. La reflexión crítica, que es una de las competencias que han de estar presentes en los profesores europeos del EEES, debería estarlo también a la hora de enjuiciar los cambios universitarios que tanto le afectan a él, a los estudiantes, a la institución y a la sociedad en general.

\section{Algunas reflexiones a modo de conclusión}

La nueva situación académica europea en el EEES, señala como necesario el perfil de un profesor académicamente versátil, potente, experto, maduro, capaz y brillante, que lea, hable y escriba en varios idiomas, un buen gestor, magnífico tutor y un buen comunicador. Deberá ser un buen representante de su universidad y mostrarse muy comprometido con el proyecto docente y trabajar mucho y bien en equipo. Es el perfil ideal de todo profesional, lo cual es más bien la expresión de un deseo que una realidad. Lo cual no impide que si Europa fuera capaz de conseguirlo, en plazo por añadidura, sólo sería motivo de aplauso y congratulación colectiva.

Si bien el proceso descrito es una inmejorable declaración de intenciones, ahora queda demostrar que si una universidad distinta es posible, sería una universidad mejor, obviamente. Pero los proyectos no siempre se cumplen, especialmente cuando existen razones muy poderosas en contra. La realidad académica española en particular, y la europea, en general, está compuesta por perfiles de profesores diversos. No todos hablan varios idiomas, no todos ellos son buenos gestores, ni excelentes comunicadores. De serlo, la persona podría elegir su tarea profesional en cual- 
quier campo. Además se le exigirá, para cumplir los requisitos de la ANECA, que sea un buen investigador, que publique con éxito y que practique algún deporte, para tener una buena forma física que le permita llegar hasta la extenuación en lo intelectual, siguiendo la cita clásica de Juvenal: "mens sana in corpore sano". Dewey (1916) habría mostrado su disconformidad con las dificultades que le aparecen al profesor/investigador con su tarea investigadora. Para él, la docencia y la investigación eran las dos caras de un mismo proceso académico, en el que habría que saber ilusionar e implicar al estudiante.

Es posible que la descripción sea tan perfecta como irreal. Los profesores universitarios europeos son personas vocacionales, habitualmente, con vicios y virtudes, con problemas personales, con una familia, con unas inquietudes, hasta con miedos y angustias. No siempre son esos seres perfectos descritos en los manuales pedagógicos. Algo similar puede suceder con los estudiantes. Los buenos estudiantes van a ser brillantes con un sistema $\mathrm{u}$ otro, pero hacer que los estudiantes graduados salgan mejor preparados para incorporarse al mercado laboral europeo no es una cuestión menor. En este sentido, la movilidad de la comunidad universitaria, tanto profesores como estudiantes, está favoreciendo mucho esta incorporación al mercado laboral europeo. El contacto personal y directo con otras realidades académicas, sociales y laborales facilita mucho la comprensión de las distintas realidades europeas así como de sus formas de trabajo y de vida.

Sólo quedaría lograr que el estudiante viviera el periodo de formación, tanto teórico como práctico con auténtico entusiasmo y máximo aprovechamiento, ya que este tiempo de formación en una u otra universidad europea sería el aprendizaje perfecto "en campo", no en las aulas ni en los laboratorios, ya que la nueva universidad demanda conocimiento teórico y práctico, tanto en ambientes artificiales (laboratorios) como naturales (realidad). ¿Se ha olvidado ya lo preconizado por Whitehead (1929) sobre la imaginación, la ilusión y los estímulos motores del conocimiento? 
El estudiante se convierte ahora en el centro de la educación superior, lo cual no quedaba del todo claro anteriormente. Ramsden (1992) ya estableció lo que para él era la estructura de las teorías de la educación: la primera, consistente en lo que el profesor enseña a los estudiantes, teoría que muestra algunas afinidades con el compromiso superficial con el contenido que tipifica una aproximación superficial; la segunda, la enseñanza como una actividad de organizar a los estudiantes, en el que el foco se aleja del profesor para enfocar al estudiante; y la tercera, contemplando la posibilidad que el profesor tiene de hacer el aprendizaje posible.

En cuanto al docente, si éste fuera tan perfecto, como se define el perfil del profesor europeo, en España los profesores más valiosos huirían a la empresa productivo-comercial-financiera privada, donde el nivel de recompensas económicas está muy por encima del académico, lo que también podría subsanarse por parte de la institución académica mediante distinto tipo de incentivos: económicos, promocionales o sociales. Es necesario añadir que los profesores universitarios que lo eran ya antes de la implantación del EEES, no han tenido la oportunidad de reflexionar, ni debatir, ni mucho menos de criticar constructivamente el proceso. El Plan Bolonia ha venido impuesto de forma vertical por las autoridades político-educativas del ámbito europeo. Como en otras ocasiones y en tantas otras cuestiones, las normas han sido escritas por tecnócratas europeos, bastante alejados de la realidad académica, que desconocen los problemas universitarios en el día-a-día. En su mayoría, los profesores ni siquiera han sido consultados y han de adaptarse en un tiempo limitado al EEES, lo que apunta un cierto carácter anti-democrático. No es de extrañar la cadena de pre-jubilaciones que se está produciendo entre el profesorado tradicional, que no está preparado para afrontar los cambios acaecidos. No están familiarizados con las TIC ni desean familiarizarse. Argumentan que si la universidad era la institución más antigua, estable, y longeva de todas, no hay razón para hacer la revolución universitaria en este momento. 
Tampoco todos los estudiantes están de acuerdo con el proceso Bolonia, a juzgar por el número de manifestaciones que se están produciendo. Condiciones como la asistencia a clase, la movilidad europea, establece grupos de ventaja frente a aquellos otros estudiantes que necesitan un apoyo económico. Los resistentes se quejan de la falta de democratización universitaria, y los estudiantes que creen en el EEES, parecen necesitar o exigir más que un tutor, un ayuda de cámara. Resulta muy cuestionable la actitud de los estudiantes actuales (eternos adolescentes) que, nacidos y acostumbrados a poseer y disfrutar de todo tipo de libertades y facilidades (lo que no siempre les ayuda a madurar ni a trabajar de una forma autónoma y responsable), que cuando abandonan la figura de protección paterna la sustituyen por el tutor universitario.

La autonomía esperada no está presente en la mayor parte de los estudiantes, que quieren seguir gozando de todos los aspectos positivos del sistema clásico, con clases magistrales, y todos los aspectos positivos del EEES, con una autorización individual y personalizada. El PDP (Desarrollo de la Planificación Personal) está muy lejos de su mentalidad y no resulta demasiado atractiva por el momento.

Mientras que con el sistema clásico español se podía hablar, a veces, de la tiranía de los profesores, en el EEES se ha llegado a hablar de la tiranía de los estudiantes. Mientras que antes eran los profesores los que evaluaban a los estudiantes, ahora son los estudiantes los que están en las mejores condiciones para evaluar al profesor.

Las aplicaciones del sistema que se están haciendo distan todavía mucho de haber alcanzado su cenit. Parece éste un periodo de transición difícil, donde hay que abandonar los viejos vicios para adquirir e incorporar las nuevas virtudes.

La formación y el reciclaje tanto intelectual como mental por parte de profesores y de estudiantes (long-life-learning) están aún por hacerse, proceso que va a exigir un gran esfuerzo, reto 
y compromiso, por parte de todos: universidades, profesores y estudiantes, si se quieren lograr los resultados óptimos. Como diría Race (2004:121): Nunca pienses en la enseñanza, siente el aprendizaje.

Por último ¿cómo está afectando el número de universidades privadas a las universidades públicas? Mientras algunos estudiantes confundan la tutorización con el clientelismo y la universidad privada lo promueva, no podrá hablarse de una excelencia académica europea, con la que sueña toda la comunidad universitaria europea, es decir, todos los países del EEES.

Hablar de la función docente, de la formación superior, y del nuevo diseño académico invita a una gran reflexión. Una parte de esta reflexión/discusión se ha presentado ya en este artículo, pero queda un buen tramo por recorrer en este largo camino (never-ending) de la docencia universitaria a ambos lados del Océano. Por el bien y el futuro de la educación superior, queda abierta una discusión por tanto para reflexiones futuras.

\section{Bibliografía}

BROWN, G. \& ATKINS; M. (1994): Effective Teaching in Higher Education. London, Routledge.

BARBERÁ, E. (2005): La evaluación de competencias complejas: la práctica del portafolio. Eucere, La Revista Venezolana de Educación, año 9, $n^{\circ}$ 31.

BENITO, A., CRUZ, A. (2005): Nuevas claves para la docencia universitaria: en el Espacio Europeo de Educación Superior, Narcea, DL, Madrid.

DEWEY, J. (1916): Education and Democracy. New York: Macmillan.

FERNÁNDEZ, A. y MAIQUES, J.M. (2001): La carpeta docente como herramienta de evaluación y mejora de la calidad de la enseñanza. En Evaluación de politicas educativas: VIII Congreso Nacional de Teoría de la educación. Pp. 86-90.

GONZÁLEZ, Julia \& WAGENAAR, Robert (2003): Tuning Educational Structures in Europe. Informe final, Universidad de Deusto.

GONZÁLEZ RAMÍREZ, T. (2005): El Espacio Europeo de Educación Superior: 
Una nueva oportunidad para la universidad. En P. Colás y J. de Pablos (Coords.): La Universidad en la Unión Europea. El Espacio Europeo de Educación superior y su impacto en la docencia (pp. 2753). Archidona (Málaga): Aljibe.

GOSLING, D. (2009): "Supporting Student learning", en Fry, H. et al.: A Handbook for Teaching and learning Higher Education: Enhancing Academic Practice. New York: Routledge. pp. 113-131.

ICE (2004): Programa de mejora e Innovación de la Docencia (En el marco de la convergencia al Espacio Europeo de Educación Superior). Univ. Zaragoza.

MARTÍNEZ SEGURA, Ma José (Coord), (2009): “Contextualización y uso del portafolios", en El portafolios para el aprendizaje, pp. 19-45. Edit. EDITUM, Univ. Murcia.

QUINTANA, Hilda E (2000): El Portafolio como estrategia para la evaluación, Editorial GRAO, Barcelona.

RACE, P. (2004): The lecturer's toolkit. A resource for Developing, Learning, Teaching and Assessment, New York: Routledge.

RAMSDEM, P. (1992): Learning to teaching in Higher Education. London: Routledge.

SANAGUSTÍN, M.V.; PUYAL, E.; MOSEÑE; J.A.; TRICAS, J.M. (2004): El Espacio Europeo de Educación superior, nuevos planes de estudio y presencia de la sociología de las organizaciones en los mismos", en Sociología de las Organizaciones. Una mirada crítica desde la Universidad, Egido Editorial, Zaragoza, pp. 67-76.

SANAGUSTÍN, M.V., CANO; J. (2005): Retos de la convergencia europea para la universidad. Universidad de Zaragoza.

TIERNEY, R.J. et al. (1991): Portfolio assessment in the reading-writing classroom. Christopher-Gordon Publishers, Norwood, MA.

TORRES, J. (1996): Globalización e interdisciplinariedad: El currículo integrado. Morata DL. Madrid.

WHITE, E. (1989): Teaching and assessing writing. Jossey-Bass Publishers, SanFrancisco.

WHITEHEAD, A.N. (1929): Process and Reality. An Essay in Cosmology, New York: Macmillan.

ZABALZA, M.A. (2004): Guía para la planificación de la docencia universitaria en el marco del EEES. Documento de Trabajo. Universidad de Santiago de Compostela. 
Repensando la universidad europea

\section{Webgrafía:}

http:/ / www.mec.es/uni/jsp/plantilla?id=3501 Fecha 8-1-2010

www.wikipedia.org/wiki/Proceso_de_Bolonia Fecha 8-1-2010 